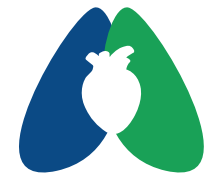

ASSOBRAFIR

C I Ê N C I A

\section{Associação da capacidade vital forçada com a endurance muscular respiratória e a capacidade funcional em pacientes com DPOC}

\author{
Association of forced vital capacity with respiratory muscle \\ endurance and functional capacity in patients with COPD
}

1Departamento de Fisioterapia, Universidade Federal da Paraíba (UFPB), João Pessoa, PB, Brasil

2Unidade de Fisioterapia, Hospital Agamenon Magalhães (HAM), Recife, PE, Brasil

${ }^{3}$ Unidade de Gestão da Pesquisa, Empresa Brasileira de Serviços Hospitalares -

EBSERH, Hospital das Clínicas, Universidade Federal de Pernambuco (UFPE), Recife, PE, Brasil

4Programa de Pós-graduação em Fisioterapia, Universidade Federal da Paraíba (UFPB), João Pessoa, PB, Brasil

Como citar: Felix JVS, Souza ED, Amaral TCN, Pedrosa R, Elihimas Júnior UF, Lima JHM, França EET. Associação da capacidade vital forçada com a endurance muscular respiratória e a capacidade funcional em pacientes com DPOC. ASSOBRAFIR Ciênc. 2022;13:e43873. https://doi. org/10.47066/2177-9333.AC.2020.0044

Submissão em: Junho 09, 2021 Aceito em: Janeiro 09, 2022

Estudo realizado em: Universidade Federal da Paraíba (UFPB), João Pessoa, PB, Brasil. Aprovação ética: CAEE

86468218.6.0000.5183 da Universidade

Federal da Paraíba, nº 2.596.531.

*Autor correspondente: Eduardo

Eriko Tenório França. E-mail:

edueriko@hotmail.com
João Victor dos Santos Felix ${ }^{1}$ (D), Emily Dias de Souza (D),

Thamara Cunha do Nascimento Amaral ${ }^{2}$ (D), Rafaela Pedrosa ${ }^{1}$ (D),

Ubiraçé Fernando Elihimas Júnior ${ }^{3}$ (1) , José Heriston de Morais Lima ${ }^{1} \mathbb{B}^{\text {, }}$

Eduardo Eriko Tenório França1,4* (i)

\begin{abstract}
Resumo
Introdução: A perda do recolhimento elástico pulmonar e a limitação ao fluxo aéreo em pacientes com DPOC estão relacionadas as alterações na biomecânica dos músculos respiratórios e na função pulmonar. A dispneia, sintoma comum nesses pacientes está associada a limitação ao exercício e redução na força e endurance muscular respiratória. Objetivo: Avaliar se há associação da capacidade vital forçada (CPF) com a endurance muscular respiratória e a capacidade funcional de pacientes DPOC. Métodos: Trata-se de um estudo analítico, observacional e transversal. A amostra foi composta por 40 pacientes com DPOC, de ambos os sexos, atendidos no ambulatório de pneumologia de um hospital público da cidade de João Pessoa, Paraíba. Os dados referentes a CVF foram extraídos através da análise de resultados da espirometria. A endurance muscular respiratória foi avaliada pelo índice de resistência a fadiga (IRF) e a capacidade funcional pelo teste de caminha de 6 minnutos (TC6). Resultados: Apenas 22,5\% dos participantes ( $n=9$ ) tinham redução na endurance muscular respiratória, porém em relação a distância percorrida no TC6, 97,4\% $(n=38)$ apresentaram uma capacidade funcional limitada. Observamos que não houve associação entre os valores da CVF com a endurance muscular respiratória $(p=0,825)$. No entanto, encontramos uma associação positiva entre a CVF com a distância percorrida no TC6 ( $p=0,012)$, demonstrando que quanto maior a CVF melhor era o desempenho no TC6. Conclusão: O presente estudo demonstrou que a redução da CVF pode ser um fator de limitação da capacidade funcional em pacientes com DPOC.
\end{abstract}

Palavras-chave: DPOC; Capacidade vital; Resistência física; Teste de caminhada.

\section{Abstract}

Background: Loss of pulmonary elastic recoil and airflow limitation in patients with COPD are related to changes in the biomechanics of respiratory muscles and lung function. Dyspnea, a common symptom in these patients, is associated with exercise limitation and reduction in respiratory muscle strength and endurance. Aim: To assess whether there is an association of forced vital capacity (FVC) with respiratory muscle endurance and functional capacity in COPD patients. Methods: This is an analytical, observational and cross-sectional study. The sample consisted of 40 patients with COPD, of both genders, treated at the pulmonology clinic of a public hospital in the city of João Pessoa, Paraíba. Data referring to FVC were extracted by analyzing the results of spirometry. Respiratory muscle endurance was assessed by the Fatigue Resistance Index (IRF) and functional capacity by the 6-minute walk test (6MWT). Results: Only $22.5 \%$ of participants $(n=9)$ reduced respiratory muscle endurance, but in relation to the distance covered in the 6MWT, 97.4\% $(n=38)$ dissipated a limited functional capacity. We observed that there was no association between FVC values and respiratory muscle resistance $(p=0.825)$. However, we found a positive association between a FVC and the distance covered on the 6MWT $(p=0.012)$, demonstrating that the higher the FVC the better the performance on the 6MWT. Conclusion: The present study alters that the reduction in FVC can be a limiting factor in functional capacity in patients with COPD.

Keywords: COPD; Vital capacity; Physical endurance; Walk test.

Este é um artigo publicado em acesso aberto (Open Access) e distribuído sob a licença Creative Commons Attribution NonComercial ShareAlike License, que permite uso, distribuição e reprodução em qualquer meio, sem restrições desde que sem fins comerciais e que o trabalho original seja corretamente citado e de forma que não indique endosso ao trabalho feito. Adicionalmente, qualquer trabalho derivado deverá ser publicado sob a mesma licença. 


\section{INTRODUÇÃO}

A doença pulmonar obstrutiva crônica (DPOC) é uma doença comum, evitável e tratável que se caracteriza pela presença de persistentes sintomas respiratórios e uma limitação progressiva de fluxo aéreo em decorrência de anormalidades (extensão da inflamação, fibrose, exsudatos no lúmen de pequenas vias aéreas) no sistema respiratório que geralmente são causadas por exposição significativa a partículas ou gases nocivos (exposições ocupacionais, incluindo poeira orgânica/inorgânica, agentes e vapores químicos; poluição do ar nas grandes cidades, entre outros)'1.

Vários fatores são responsáveis pela limitação funcional em pacientes com DPOC, porém, têm papel de destaque a limitação ventilatória e a força muscular periférica2,3. Na DPOC, a perda do recolhimento elástico pulmonar e a limitação ao fluxo aéreo progressivo promovem aprisionamento aéreo e hiperinsuflação, com aumento da capacidade residual funcional e diminuição da capacidade vital forçada (CVF). Esta hiperinsuflação favorecerá a desvantagem mecânica dos músculos inspiratórios e a redução do limiar ventilatório que irá favorecer a menor tolerância ao exercício, redução da força muscular periférica e aumento da dispneia ${ }^{4}$.

A dispneia é o sintoma mais notável que está associado a limitação ao exercício, caracterizada como uma sensação de desconforto respiratório de origem multifatorial, mas que tem sido relacionada a desvantagens mecânicas e disfunções da musculatura respiratória como a redução da força e endurance muscular respiratória ${ }^{5}$. A redução da endurance muscular respiratória é uma importante manifestação da DPOC, estando a mesma função muscular prejudicada temos redução da tolerância ao exercício, da qualidade de vida relacionada a saúde (QVRS) e da sobrevida ${ }^{6}$.

São poucos os estudos que associam os valores de CVF, com a endurance muscular respiratória e a capacidade funcional desses pacientes. No entanto, necessitamos de mais estudos que façam uma correlação específica entre essas variáveis, de forma que possamos identificar se os valores de CVF podem servir como marcadores funcionais para predizer o comprometimento funcional do paciente com DPOC. Diante disso, o presente estudo tem como objetivo avaliar a associação entre a medida de CVF com a endurance muscular respiratória e a capacidade funcional de pacientes DPOC.

\section{MÉTODOS}

Trata-se um estudo analítico, observacional e transversal. A amostra foi composta por 40 pacientes com DPOC, de ambos os sexos, atendidos no ambulatório de pneumologia de um hospital público da cidade de João Pessoa, Paraíba. A participação de todos ocorreu de forma voluntária através da assinatura de um Termo de Consentimento Livre e Esclarecido, devidamente elaborado de acordo com a Resolução $n^{\circ} 466 / 12$ do Conselho Nacional de Saúde, que trata das diretrizes e normas de pesquisas envolvendo seres humanos. $O$ estudo foi conduzido de acordo com os princípios da declaração de Helsinki. Este estudo é parte integral do projeto de pesquisa intitulado "Prevenção, avaliação e terapêutica da reabilitação nas desordens cardiovasculares, respiratórias e metabólica", do Departamento de Fisioterapia da Universidade Federal da Paraíba, com o número do CAAE 86468218.6.0000.5183 e Registro Brasileiro de Ensaio Clínicos (ReBEC) de número RBR-89QMHC, aprovado pelo Comitê de Ética e Pesquisa (CEP) do Hospital Universitário Lauro Wanderley.

Foram considerados como critérios de inclusão no estudo pacientes com diagnóstico de DPOC e espirometria que comprovasse a obstrução irreversível, de acordo com a estratificação de gravidade da Global Initiative for Chronic Obstructive Lung Disease (GOLD) Reports 2021', maiores de 18 anos, de ambos os sexos e já pertencentes ao ambulatório de pneumologia ou que foram admitidos durante o período do estudo.

Foram excluídos do estudo pacientes com dificuldade de compreender a avaliação espirométrica e a avaliação da força muscular respiratória; portadores de doenças neurodegenerativas incapazes de realizar as mesmas; instabilidade clínica prévia há menos de seis meses ou no dia da avaliação; qualquer tipo de doença cardíaca ou outra doença respiratória comprovada por exames de imagem ou laboratoriais; problemas ortopédicos e/ou que influenciassem na marcha; pacientes com índice de massa corpórea (IMC) $>35 \mathrm{Kg} / \mathrm{m}^{2}$; problemas psicológicos descompensados; que se recusassem a participar da pesquisa ou que estavam uso de oxigênio suplementar.

Para o recrutamento dos pacientes a fim de integrálos a amostra, foram realizadas análises dos registros médicos, através das informações demográficas, história clínica e diagnóstico. Os pacientes selecionados, de acordo com os critérios de inclusão e exclusão do estudo, receberam o convite para a participação por telefone e foram agendados para a avaliação. Antes de iniciar a avaliação, os testes a serem realizados foram descritos de forma detalhada, sendo esclarecida quaisquer dúvidas.

Os dados referentes a CVF foram extraídos através da análise de resultados da espirometria, de acordo com Graham et al.7. O teste foi realizado seguindo os critérios estabelecidos pela GOLD', onde o paciente colocava a boca no bocal e o técnico garantia que não houvesse vazamento. O paciente foi orientado a realizar uma inspiração máximo de ar, com uma pausa de menos de 1s na capacidade pulmonar total e em seguida realizar uma expiração forçado por pelo menos 6 segundos. Foram incluídos resultados de espirometria de seis meses anteriores ao estudo. Através dos resultados da espirometria foram obtidos os valores da CVF, volume expiratório forçado no primeiro segundo (VEF1) e a relação VEF1/CVF'. 
Para obtenção dos dados referentes a variável da endurance muscular respiratória foi considerado os dados obtidos pelo teste de carga incremental, onde os pacientes foram instruídos a realizar o maior número de respirações, expirar até o volume residual (VR) e logo após, fazer uma inspiração máxima com uma carga inicial de $10 \mathrm{cmH}_{2} \mathrm{O}$, por um período de 2 minutos, sem remover o equipamento da boca ${ }^{9}$. Na avaliação da função muscular respiratória foi utilizado o dispositivo eletrônico computadorizado (KH2; Power Breathe International Ltd. UK) sendo usado o software Breathelink como feedback visual. Logo após o primeiro ciclo, foi dado 1 minuto de descanso e um novo ciclo de 2 minutos se iniciou, com um acréscimo de mais $10 \mathrm{cmH}_{2} \mathrm{O}$, somando um total de $20 \mathrm{cmH}_{2} \mathrm{O}$. A cada novo ciclo, foram acrescentados mais $10 \mathrm{cmH}_{2} \mathrm{O}$ a carga. Quando observada falha, incapacidade de vencer a carga ou sinais de dispneia ou fadiga, o teste foi interrompido.

Foi considerada a maior carga sustentada por pelo menos 1 minuto como o valor da pressão inspiratória máxima (PImáx) sustentada. O tempo começou a ser contado após o paciente acoplar a boca no equipamento e realizar a primeira respiração e a contagem só parou quando o mesmo retirou o dispositivo da boca com a interrupção do teste, o tempo total foi registrado.

A PImáx foi avaliada antes e imediatamente após o teste de carga incremental para verificar a ocorrência de fadiga muscular através do índice de resistência a fadiga (IRF), referente a divisão entre a PImáx final e inicial, quando o resultado é menor que $88 \%$ considera-se um déficit na resistência da musculatura respiratória ${ }^{10}$.

Para a variável capacidade funcional, foi realizado o teste de caminhada de 6 minutos (TC6), estando de acordo com a American Thoracic Society - ATS ${ }^{11}$. O teste tem como objetivo determinar a tolerância ao exercício e a saturação periférica de oxigênio $\left(\mathrm{SpO}_{2}\right)$ durante um exercício submáximo, avaliando a capacidade funcional do indivíduo. Ele avalia a distância máxima que o paciente consegue percorrer sobre uma superfície plana e rígida de 30 metros, delimitada de forma clara e visível com a utilização de 2 cones, durante o período de 6 minutos. Os pacientes foram instruídos a caminhar o mais rápido possível durante o tempo de 6 minutos sem parar. A pausa foi permitida, no entanto, o tempo continuou sendo cronometrado e ao final dos 6 minutos o teste foi finalizado. Entretendo, devido as condições físicas do paciente, o teste foi realizado uma única vez. Ao final foi registrado a distância percorrida, sendo utilizado como referência para a distância predita a fórmula validada para a população brasileira ${ }^{12}$. Os participantes que percorreram a distância predita foram incluídos no grupo de capacidade funcional normal e os que não percorreram no de capacidade funcional limitada. Para calcular os valores previstos no TC6 foram utilizadas as seguintes fórmulas:

Distância percorrida no TC6 $=299,296-(2,728$ vs. idade $)-(2,160$ vs. peso $)+(361,731$ vs. altura $)+(56,386$ vs. gênero); erro padrão da estimativa $=57,7 \mathrm{~m}^{12}$.

$O$ banco de dados foi elaborado no programa EPI INFO, versão 3.5.4, onde foi realizada a validação. Após a validação o banco foi exportado para o software SPSS, versão 18 , onde foi realizado a análise. Para testar a suposição de normalidade das variáveis envolvidas no estudo foi utilizado o Teste Kolmogorov-Smirnov. Para testar as correlações da CVF com a endurance muscular respiratória e TC6 foi utilizado o Coeficiente de Correlação de Pearson. Foi levada em consideração relevância significativa de 0,05.

\section{RESULTADOS}

A Figura 1 mostra o fluxograma do presente estudo, onde inicialmente foram recrutados 115 pacientes diagnosticados com DPOC, sendo 64 excluídos. Desses

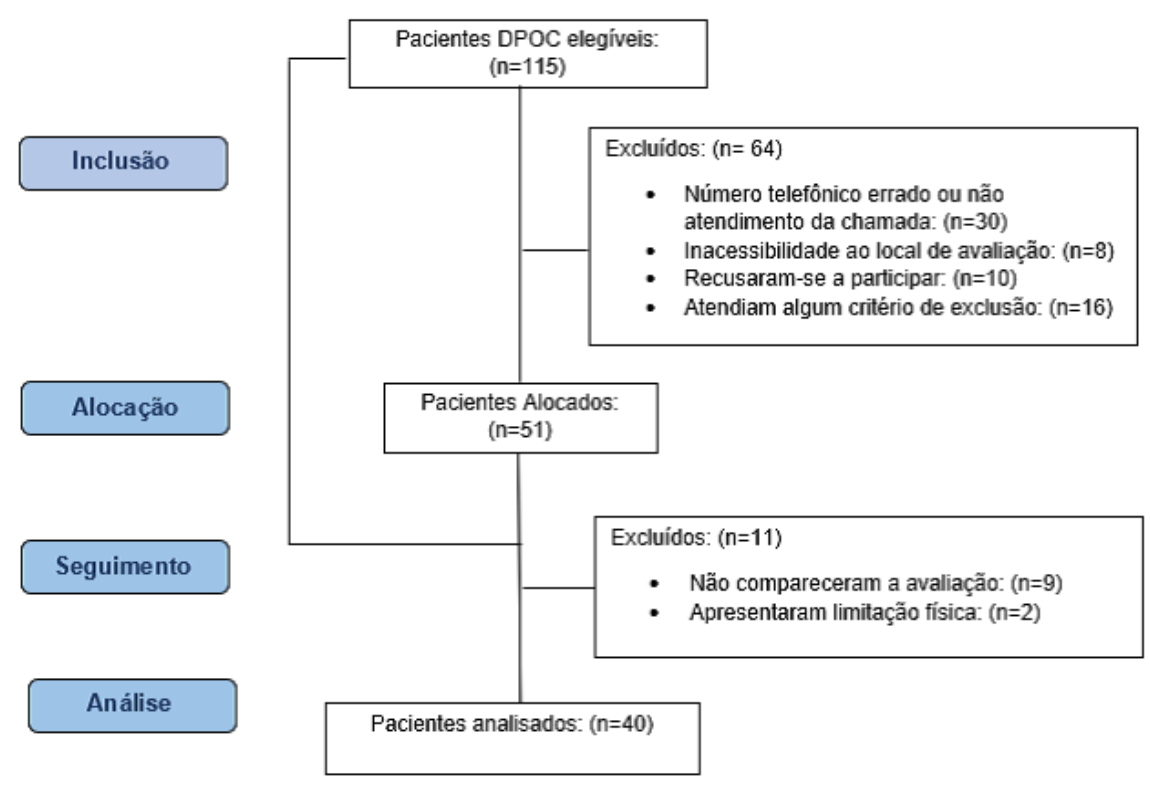

Figura 1. Fluxograma. 
64 pacientes, 30 não participaram da pesquisa por dificuldade de encontrá-los através dos contatos repassados, 8 por inacessibilidade ao local de avaliação, 10 por se recusarem em participar da pesquisa, 1 por ter sido informado óbito durante o contato inicial, 14 por relatarem sinais e sintomas de exacerbação e/ou estarem hospitalizados e o último por relato de diagnóstico de doença neoplásica pulmonar. Foram incluídos 51 pacientes, entretanto, 9 não compareceram a avaliação e 2 apresentaram incapacidade de realizar as avaliações do estudo, totalizando 40 pacientes analisados.

A Tabela 1 apresenta a caracterização da amostra, a média de idade dos participantes caracteriza uma amostra

Tabela 1. Caracterização da amostra.

\begin{tabular}{|c|c|c|c|}
\hline Variáveis & $\mathbf{N}$ & $\%$ & Média \pm DP \\
\hline Idade & & & $65,7 \pm 9,59$ \\
\hline 43 a 59 anos & 7 & 17,5 & \\
\hline 60 a 87 anos & 33 & 82,5 & \\
\hline \multicolumn{4}{|l|}{ Sexo } \\
\hline Feminino & 22 & 55,0 & \\
\hline Masculino & 18 & 45,0 & \\
\hline VEF1/CVF & & & $24 \pm 27$ \\
\hline VEF1/CVF (\%) previsto & & & $30,25 \pm 34,03$ \\
\hline CVF (L)* & & & $2,02 \pm 0,74$ \\
\hline CVF (\%) previsto & & & $64,33 \pm 23,56$ \\
\hline VEF1 (L) & & & $0,49 \pm 0,20$ \\
\hline VEF1 (\%) previsto & & & $19,29 \pm 7,87$ \\
\hline Endurance (\%) & & & $104,88 \pm 25,35$ \\
\hline TC6 $(n=39) *$ & & & $333,88 \pm 117,14$ \\
\hline TC6 (\%) previsto & & & $58,73 \pm 20,60$ \\
\hline
\end{tabular}

a)

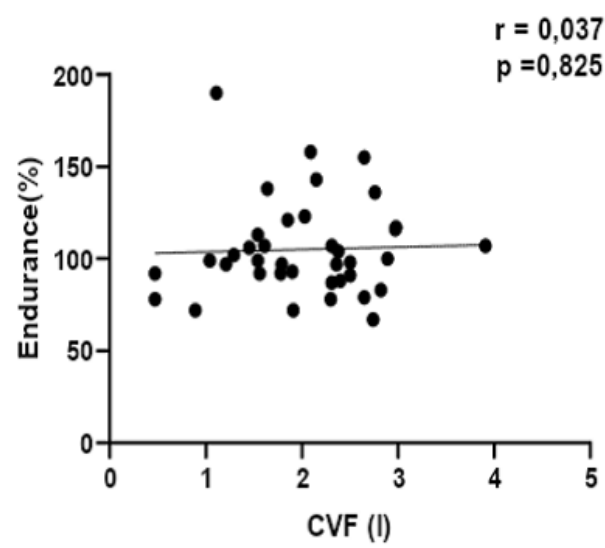

majoritariamente idosa $(65,7 \pm 9,59)$, com um maior número de participantes do sexo feminino, e também mostra a média de valores relacionados à relação VEF1/ CVF, VEF1, CVF, endurance muscular respiratória e a distância percorrida no TC6 obtidas através da avaliação dos participantes.

A Tabela 2 caracteriza os pacientes em relação aos valores da endurance muscular respiratória, demonstrando que apenas $22,5 \%$ dos participantes $(n=9)$ tinham redução na endurance muscular respiratória, porém em relação a distância percorrida no TC6, 97,4\% (n=38) dos pacientes avaliados apresentavam uma capacidade funcional limitada.

A Figura 2, traz dois gráficos de correlação entre as variáveis, CVF e endurance muscular respiratória (Figura 2a) e CVF e distância percorrida no TC6 (Figura 2b). Na Figura 2a, podemos observar que não foi encontrada correlação entre os valores de CVF e a endurance muscular respiratória ( $p=0,825)$. Já na Figura $2 b$, podemos observar correlação positiva entre a variável CVF e a distância percorrida no TC6 ( $p=0,012)$, demonstrando que quanto maior o valor da CVF da população estudada melhor era o seu desempenho no TC6.

Tabela 2. Caracterização da amostra em relação a endurance e capacidade funcional.

\begin{tabular}{cccc}
\hline Variáveis & $\mathbf{n}$ & $\%$ & p-valor \\
\hline IRF (\%) & & & \\
$>88$ & 31 & 77,5 & 0,001 \\
$<88$ & 9 & 22,5 & \\
Distância percorrida no TC6 (min) & & & \\
Capacidade funcional normal & 1 & 2,6 & $<0,001$ * \\
Capacidade funcional limitada & 38 & 97,4 & \\
\hline
\end{tabular}

IRF = índice de resistências a fadiga; TC6 = teste de caminhada de 6 minutos. ${ }^{*} \mathrm{O}$ número total de elementos não coincide com o tamanho da amostra pois um participante se negou a realizar o TC6.

b)

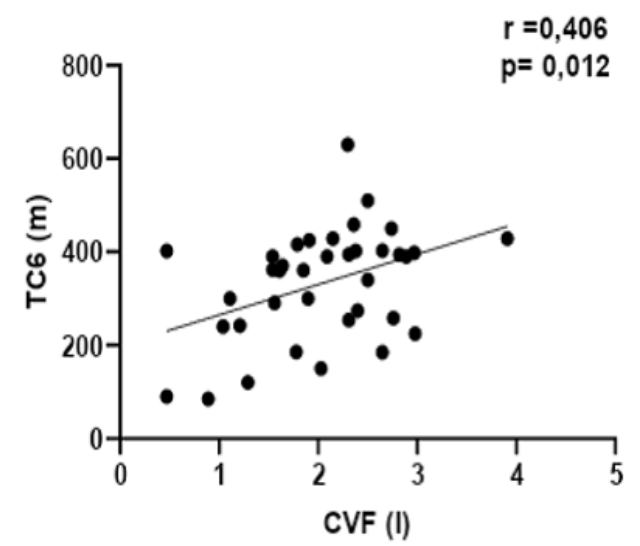

Figura 2. (a) Correlações entre a capacidade vital forçada (CVF) e endurance muscular respiratória e (b) CVF e teste de caminhada de seis minutos (TC6). 


\section{DISCUSSÃO}

Nossa pesquisa demonstrou associação positiva entre a CVF e a capacidade funcional de pacientes com DPOC, avaliada pelo TC6, demonstrando que quanto maior o valor da CVF observada na espirometria, melhor a capacidade funcional desses pacientes. No entanto, para mesma população estudada, não foi possível observar associação entre a CVF e a endurance muscular respiratória.

Nosso resultado corrobora com o estudo Moon et al. ${ }^{13}$ que realizaram um estudo transversal e avaliaram 1.386 pacientes com DPOC de leve a muito grave e identificaram que os pacientes que possuíam menores alcances no TC6 possuíam uma CVF significativamente reduzida. Isso implica que pacientes com maior gravidade expressa pela redução da CVF, atingiam uma distância percorrida no TC6 mais curta e também mostravam pontuações mais altas no índice BODE do que os pacientes que mantiveram a CVF preservada. O que os leva a concluir que a CVF reduzida seria um bom preditor para uma redução da distância percorrida no TC6 e a CVF preservada pode indicar uma melhor capacidade funcional mesmo em pacientes graves ${ }^{13}$.

De acordo com Aalstad et al. ${ }^{14}$, o TC6 é amplamente usado na avaliação da capacidade funcional na DPOC e a distância percorrida no TC6 se correlaciona bem com a dispneia, hiperinsuflação pulmonar e o grau de obstrução. Eles realizaram um estudo de coorte prospectivo que incluiu 389 pacientes de 40 a 75 anos de idade diagnosticados com DPOC e estáveis, onde foi constatado que a redução da CVF se correlacionou com mais exarcebações, declínio na distância percorrida no TC6 e consequentemente uma pior capacidade funcional ${ }^{14}$.

Segundo Perez et al. ${ }^{15}$ ao analisarem os determinantes de uma curta distância percorrida no TC6 e a queda na saturação periférica de oxigênio $\left(\mathrm{SpO}_{2}\right)$ induzida por exercício, eles procuraram avaliar a influência da idade, sexo, obesidade, baixo IMC, escala de dispneia (mMRC), os percentuais (\%) previsto de VEF1 e CVF, hiperinsuflação e doenças cardiovasculares. Como principais resultados, foi mostrado que a CVF, assim como a idade, a escala de dispneia e a $\mathrm{SpO}_{2}$, foram os principais determinantes para um mau desempenho no TC6 ${ }^{15}$.

Em um outro estudo transversal com 70 pacientes com DPOC estável, foi levantado a hipótese de que a quantidade de passos no TC6 também seja um eficiente indicador de uma capacidade funcional reduzida, o principal resultado deste estudo é que a quantidade de passos no TC6 mostrou uma boa correlação com a função pulmonar e a qualidade de vida dos pacientes, o que trouxe o pressuposto de que essa outra forma de se avaliar através do TC6 seja um novo índice de capacidade funcional e em alguns casos, um preditor melhor que a distância percorrida no TC6 ${ }^{16}$.

$\mathrm{Na}$ atual amostra estudada não foi possível observar associação da CVF com a endurance muscular respiratória. Já Formiga et al. ${ }^{17}$ ao estudarem 81 pacientes do sexo masculino com DPOC de leve a muito grave, e avaliarem a endurance muscular respiratória através do teste de resistência incremental, identificaram que a redução da endurance esteve presente em toda a amostra, estando mais comprometida em indivíduos com valores mais baixos de VEF1 que caracterizam maior gravidade da doença.

No entanto, Singh et al. ${ }^{18}$ trazem que apesar da hiperinsuflação pulmonar e a baixa resistência ao exercício serem fatores importantes na DPOC e pareçam estar relacionados, a correlação permanece incerta. Uma hipótese é a de que a hiperinsuflação estática e dinâmica se relacionem de formas diferentes com a endurance da musculatura respiratória, onde a hiperinsuflação dinâmica seria um melhor preditor para a melhora na tolerância ao exercício, o que poderia explicar a falta de associação entre a CVF e a endurance muscular respiratória em nossa amostra estudada, uma vez que a avaliação da endurance foi realizada inteiramente de forma estática, sem que houvesse nenhum tipo de exercício antes de sua avaliação ${ }^{18}$.

Ainda, deve-se notar que os mecanismos subjacentes à intolerância ao exercício são complexos e multifatoriais, a fadiga muscular juntamente com a dispneia contribuem significativamente para a limitação da resistência ao exercício, mas a forma que esses fatores contribuem individualmente é difícil de determinar ${ }^{18}$.

Liu et al. ${ }^{19}$ ao recrutarem 88 pacientes com DPOC estável e analisarem a função muscular respiratória e sua associação com a força e resistência da musculatura periférica observaram que a força de membros superiores se correlaciona bem com a função pulmonar e da musculatura respiratória desses pacientes. Músculos proximais na região do tórax, cintura escapular e ombros fazem parte da musculatura respiratória acessória e desempenham um papel importante na respiração uma vez que os músculos principais neste processo (diafragma e intercostais externos) estejam disfuncionais ${ }^{19}$.

Uma limitação deste estudo refere-se a baixa aderência dos participantes, visto que o ambulatório de Pneumologia atende a grande maioria dos pacientes que residem fora da cidade onde foram realizadas as avaliações. Além disso, devido ao perfil dos pacientes recrutados, não foi possível realizar o teste de caminhada mais de uma vez para diminuir os impactos que o efeito aprendizagem pode exercer sobre o teste.

\section{CONCLUSÃO}

Em conclusão, o presente estudo demonstrou que a redução da CVF pode ser um fator de limitação da capacidade funcional em pacientes com DPOC. Evidenciamos também que na amostra estudada não foi encontrada associação entre a CVF e a endurance muscular respiratória. 


\section{FONTE DE FINANCIAMENTO}

Nada a declarar.

\section{CONFLITO DE INTERESSES}

Nada a declarar.

\section{REFERÊNCIAS}

1. GOLD: Global Initiative for Chronic Obstructive Lung Disease. Global strategy for the diagnosis, management, and prevention of COPD (GOLD 2021 Reports) [Internet]. 2021 [citado em 2021 Jun 9]. Disponível em: https:// goldcopd.org/wp-content/uploads/2020/11/GOLD-REPORT2021-v1.1-5Nov20_WMV.pdf

2. American Thoracic Sociey, European Respiratory Society. Skeletal muscle dysfunction in chronic obstructive pulmonary disease. A statement of the American Thoracic Society and European Respiratory Society. Am J Respir Crit Care Med. 1999;159(4 Pt 2):S1-40. http://dx.doi.org/10.1164/ ajrccm.159.supplement_1.99titlepage. PMid:10194189.

3. O'Donnell D, Revill S, Webb K. Dynamic hyperinflation and exercise intolerance in chronic obstructive pulmonary disease. Am J Respir Crit Care Med. 2001;164(5):770-7. http:// dx.doi.org/10.1164/ajrccm.164.5.2012122. PMid:11549531.

4. Rodrigues J Jr, Pereira C. Resposta a broncodilatador na espirometria: que parâmetros e valores são clinicamente relevantes em doenças obstrutivas? J Pneumol. 2001;27(1):3547. http://dx.doi.org/10.1590/S0102-35862001000100007.

5. Charususin N, Dacha S, Gosselink R, Decramer M, Von Leupoldt A, Reijnders $T$, et al. Respiratory muscle function and exercise limitation in patients with chronic obstructive pulmonary disease: a review. Expert Rev Respir Med. 2018;12(1):67-79. http://dx.doi.org/10.1080/17476348.201 8.1398084. PMid:29072087.

6. Ryrso $C K$, Thaning $P$, Siebenmann $C$, Lundby $C$, Lange $P$, Pedersen $B$, et al. Effect of endurance versus resistance training on local muscle and systemic inflammation and oxidative stress in COPD. Scand J Med Sci Sports. 2018;28(11):2339-48. http://dx.doi.org/10.1111/sms.13227. PMid:29802649.

7. Graham BL, Steenbruggen I, Miller MR, Barjaktarevic IZ, Cooper BG, Hall GL, et al. Standardization of Spirometry 2019 Update. An Official American Thoracic Society and European Respiratory Society Technical Statement. Am J Respir Crit Care Med. 2019 Out 15;200(8):e70-88. http:// dx.doi.org/10.1164/rccm.201908-1590ST. PMid:31613151.

8. Lamb K, Theodore D, Bhutta B. Spirometry. Treasure Island (FL): Stat Pearls Publishing; 2020.
9. Dias F, Sampaio L, Silva G, Gomes E, Nascimento E, Alves, $V$ et al. Home-based pulmonar rehabilitation in patients with chronic obstructive pulmonar disease: a randomized clinical trial. 2013;537-44. http://dx.doi.org/ 10.2147/COPD.S50213..

10. Chang AT, Boots R, Brown M, Paratz J, Hodges P. Reduced inspiratory muscle endurance following successful weaning from prolonged mechanical ventilation. Chest. 2005;128(2):553-9. http://dx.doi.org/10.1016/S00123692(15)50395-4. PMid:16100137.

11. AST: American Thoracic Society. ATS statement: guidelines for the six-minute walk test. Am J Respir Crit Care Med. 2002;166(1):111-7. http://dx.doi.org/10.1164/ ajrccm.166.1.at1102. PMid:12091180.

12. Dourado VZ, Vidotto MC, Guerra RL. Reference equations for the performance of healthy adults on field walking tests. J Bras Pneumol. 2011 Set-Out;37(5):607-14. http://dx.doi. org/10.1590/S1806-37132011000500007. PMid:22042392.

13. Moon SM, Lim JH, Hong YS, Shin KC, Lee CY, Kim DJ, et al. Clinical impact of forced vital capacity on exercise performance in patients with chronic obstructive pulmonary disease. J Thorac Dis. 2021 Fev;13(2):837-46. http://dx.doi. org/10.21037/jtd-20-1098a. PMid:33717557.

14. Aalstad LT, Hardie J, Espehaug B, Thorsen E, Bakke P, Eagan $T$, et al. Lung hyperinflation and functional exercise capacity in patients with COPD - A three year longitudinal study. BMC Pulm Med. 2018;18(1):187. http://dx.doi.org/10.1186/ s12890-018-0747-9. PMid:30522466.

15. Perez $T$, Deslée $G$, Burgel $P$, Caillaud D, Rouzic O, Zysman $M$ et al. Predictors in routine practice of 6-min walking distance and oxygen desaturation in patients with COPD: impact of comorbidities. Int J Chron Obstruct Pulmon Dis. 2019;14:1399-410. http://dx.doi.org/10.2147/COPD.S188412.

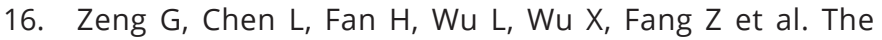
relationship between steps of 6MWT and COPD severity: a cross-sectional stuy. Int J Chron Obstruct Pulmon Dis. 2019;14:141-8. http://dx.doi.org/10.2147/COPD.S188994.

17. Formiga M, Roach K, Vital I, Urdaneta G, Balestrini K, Calderon-Candelario $\mathrm{R}$ et al. Reliability and validity of the test of incremental respiratory endurance measures of inspiratory muscle performance in COPD. Int J Chron Obstruct Pulmon Dis. 2018;13:1569-76. http://dx.doi.org/10.2147/COPD.S160512.

18. Singh S, Maltais F, Tombs L, Fahy W, Vahdati-Bolouri M, Locantore $\mathrm{N}$ et al. Relationship between exercise endurance and static hyperinflation in a post hoc analysis of two clinical trials in patients with COPD. Int J Chron Obstruct Pulmon Dis. 2018;13:203-15. http://dx.doi.org/10.2147/COPD.S145285.

19. Liu X, Li P, Wang Z, Lu Y, Li N, Xiao L et al. Evaluation of isokinetic muscle strength of upper limb and the relationship with pulmonary function and respiratory muscle strength in stable COPD patients. Int J Chron Obstruct Pulmon Dis. 2019;14:2027-36. http://dx.doi.org/10.2147/COPD.S214737. 